\title{
A Novel Triple-Mode Bandpass Filter Based on a Dual-Mode Defected Ground Structure Resonator and a Microstrip Resonator
}

\author{
Xuehui Guan, ${ }^{1,2}$ Ye Yuan, ${ }^{1}$ Lan Song, ${ }^{1}$ Xiaoyan Wang, ${ }^{1}$ \\ Jiuhuai Lei, ${ }^{1}$ Lei Zhu, ${ }^{1}$ and Peng Cai ${ }^{3}$ \\ ${ }^{1}$ School of Information Engineering, East China Jiaotong University, Nanchang 330013, China \\ ${ }^{2}$ State Key Laboratory of Millimeter Waves, Southeast University, Nanjing 210096, China \\ ${ }^{3}$ School of Software and Communication Engineering, Jiangxi University of Finance and Economics, \\ Nanchang 330032, China
}

Correspondence should be addressed to Xuehui Guan; xuehuiguan@yahoo.com.cn

Received 21 February 2013; Accepted 25 March 2013

Academic Editor: Haiwen Liu

Copyright (C) 2013 Xuehui Guan et al. This is an open access article distributed under the Creative Commons Attribution License, which permits unrestricted use, distribution, and reproduction in any medium, provided the original work is properly cited.

\begin{abstract}
A novel triple-mode bandpass filter (BPF) using a dual-mode defected ground structure (DGS) resonator and a microstrip resonator is proposed in this paper. The dual-mode characteristic is achieved by loading a defected T-shaped stub to a uniform impedance DGS resonator. A uniform impedance microstrip resonator is designed on the top layer of the DGS resonator and a compact bandpass filter with three resonant modes in the passband can be achieved. A coupling scheme for the structure is given and the coupling matrix is synthesized. Based on the structure, a triple-mode BPF with central frequency of $2.57 \mathrm{GHz}$ and equal ripple bandwidth of $15 \%$ is designed for the Wireless Local Area Network. Three transmission zeros are achieved at $1.48 \mathrm{GHz}, 2.17 \mathrm{GHz}$, and $4.18 \mathrm{GHz}$, respectively, which improve the stopband characteristics of the filter. The proposed filter is fabricated and measured. Good agreements between measured results and simulated results verify the proposed structure well.
\end{abstract}

\section{Introduction}

Recent decades have seen the rapid development of wireless technology; as a result, there is an increasing demand on high performance microwave filters [1]. The appearance of dualmode resonators has found its way into the application of filters and gained increasing popularity among the microwave filters for their capability to make a reduction of the numbers of resonating components. Since a microstrip ring dual-mode bandpass filter (BPF) was firstly proposed by Wolff in 1972 [2], various forms of dual-mode microstrip resonators and filters have been reported, including square patch resonators [3], square loop resonators [4], triangular loop resonators [5], and hexagonal loop resonators [6]. Degenerate modes are excited by the perturbations within dual-mode resonators. Dualmode or triple-mode characteristics can also be achieved by loading a stub to a resonator [7]. When changing the size of loading stub, the even-mode resonant frequencies can be easily controlled whereas the odd-mode resonant frequencies keep almost unchanged. Recently, microwave circuits are popularly designed on the ground plane [8-10], such as slotline with defected ground structure (DGS) stubs [11], defected resonator [12], and defected stepped impedance resonator [13]. It provides a novel way for realizing dual-mode filters and multimode filters by fully utilizing the printed circuit board. Multimode filters can also be realized by using dual-mode resonator doublets [14]. In our previous work [15], a four-mode BPF is achieved by combination of two dual-mode microstrip resonators. By setting two stub-loaded dual-mode resonators in parallel, a BPF with four poles is realized. However, the resonators could be properly arranged to minimize the circuit size. 
In this paper, a novel triple-mode bandpass filter is proposed using a dual-mode defected structure resonator and a microstrip resonator. A coupling scheme for the filter is given and the coupling matrix is synthesized. The ideal response of the filter agrees well with the simulated results. Compared with traditional filter of the same characteristic, the size of proposed filter reduces approximately $2 / 3$. Three transmission zeros are achieved at $1.48 \mathrm{GHz}, 2.17 \mathrm{GHz}$, and $4.18 \mathrm{GHz}$ in the stopband of the filter, which greatly improve the selectivity and rejection of the filter. The proposed BPF is simulated, implemented, and measured. Good agreement is observed between simulated results and measured results.

\section{Theoretical Analysis}

2.1. Analysis of Filter Structure. Figure 1 is the 3D structure of the proposed triple-mode filter. The filter structure can be divided into three layers: the top layer is covered with a microstrip open-loop resonator and a pair of microstrip feed lines, the middle layer is the substrate, and the bottom layer is metal ground that is loaded by a dual-mode DGS resonator. The microstrip resonator on the top layer and the DGS resonator in the bottom layer are both directly coupled to the microstrip feed lines. Since the microstrip resonator and the DGS resonator are located on different layer of the circuit, the space of the circuit is fully utilized and the size of filter is reduced.

The coupling scheme of the triple-mode filter is presented in Figure 2. The dark circles and the white circles indicate resonant modes of resonators and source/load, respectively. Mode 1 is generated by the microstrip resonator; modes 2 and 3 are even and odd modes of the dual-mode DGS resonator [16]. These modes are all directly coupled to both the source and the load. The coupling between microstrip resonator and source/load can be modified by changing their distance and overlap length. The coupling between dual-mode resonator and source/load also can be tuned by changing the location of the resonator. Commonly, the coupling between the microstrip resonator and source or load and the coupling between the even mode of the dualmode DGS resonator and input or output are all positive. The coupling between the odd mode of the dual-mode DGS resonator and source is positive, while the coupling between this mode and load is negative. The dashed line indicates the coupling between source and load that is determined by the gap between input and output microstrip line. Therefore, the corresponding coupling matrix of the coupling scheme is given by

$$
M=\left[\begin{array}{ccccc}
0 & M_{S 1} & M_{S 2} & M_{S 3} & M_{S L} \\
M_{1 S} & M_{11} & 0 & 0 & M_{1 L} \\
M_{2 S} & 0 & M_{22} & 0 & M_{2 L} \\
M_{3 S} & 0 & 0 & M_{33} & M_{3 L} \\
M_{L S} & M_{L 1} & M_{L 2} & M_{L 3} & 0
\end{array}\right]
$$

Due to symmetrical geometry of the proposed filter, the coupling coefficients agree with $M_{S 1}=M_{L 1}, M_{S 2}=M_{L 2}$, and $M_{S 3}=-M_{L 3}$. A transmission zero is produced by the coupling between source and load which improve the

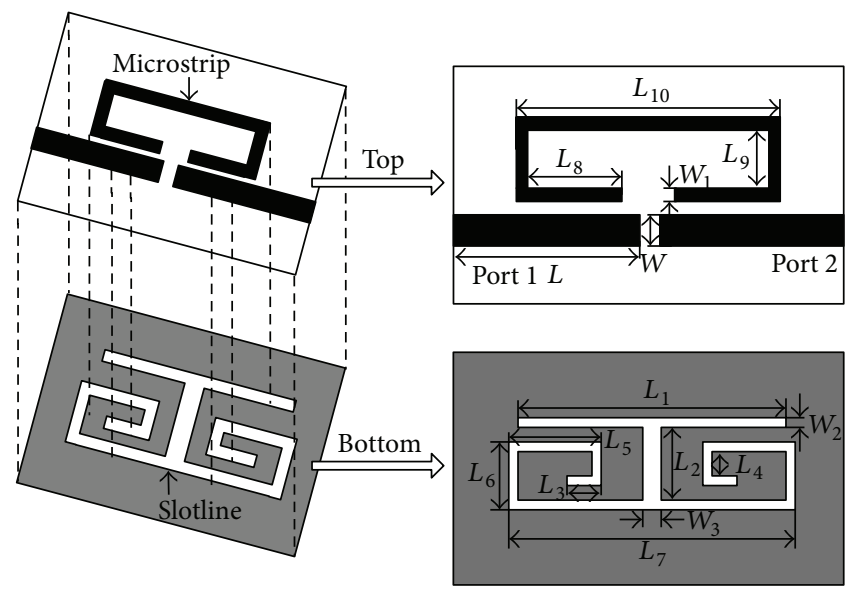

FIGURE 1: Configuration of slotline-microstrip triple-mode BPF.

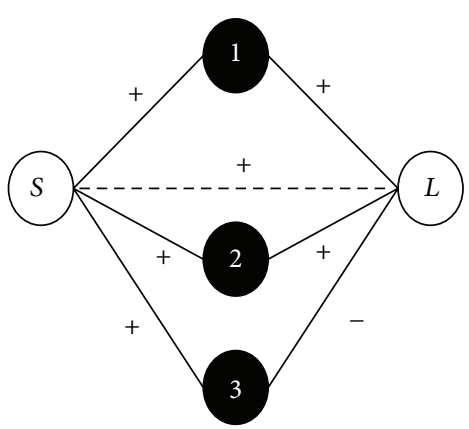

Figure 2: The coupling scheme of proposed triple-mode BPF.

selectivity of the proposed BPF. Therefore, the generalized coupling matrix for the proposed BPF with central frequency of $2.57 \mathrm{GHz}$ can be obtained on the basis of the approach of synthesis in [17] as follows:

$$
M=\left[\begin{array}{ccccc}
0 & 0.6369 & 0.3645 & 0.7824 & 0.0282 \\
0.6369 & -1.5130 & 0 & 0 & 0.6369 \\
0.3645 & 0 & 1.3070 & 0 & 0.3645 \\
0.7824 & 0 & 0 & 0.5266 & -0.7824 \\
0.0282 & 0.6369 & 0.3645 & -0.7824 & 0
\end{array}\right] .
$$

The synthesized scatting characteristic of the proposed filter is shown in Figure 3. The solid line and the dashed line indicate insertion loss and return loss, respectively. Three transmission poles are clearly observed in the passband of the filter. In addition, three transmission zeros are created at $1.41 \mathrm{GHz}, 2.17 \mathrm{GHz}$, and $4.18 \mathrm{GHz}$, which improve the selectivity in the transition band and attenuation in the stopband. The return loss in the passband is larger than $20 \mathrm{~dB}$ and the minimum insertion loss in the stopband is almost greater than $20 \mathrm{~dB}$.

2.2. Analysis of Transmission Zeros. Figure 4 shows the transmission characteristics of the filter versus the length of microstrip feed line. When the length of microstrip feed line 


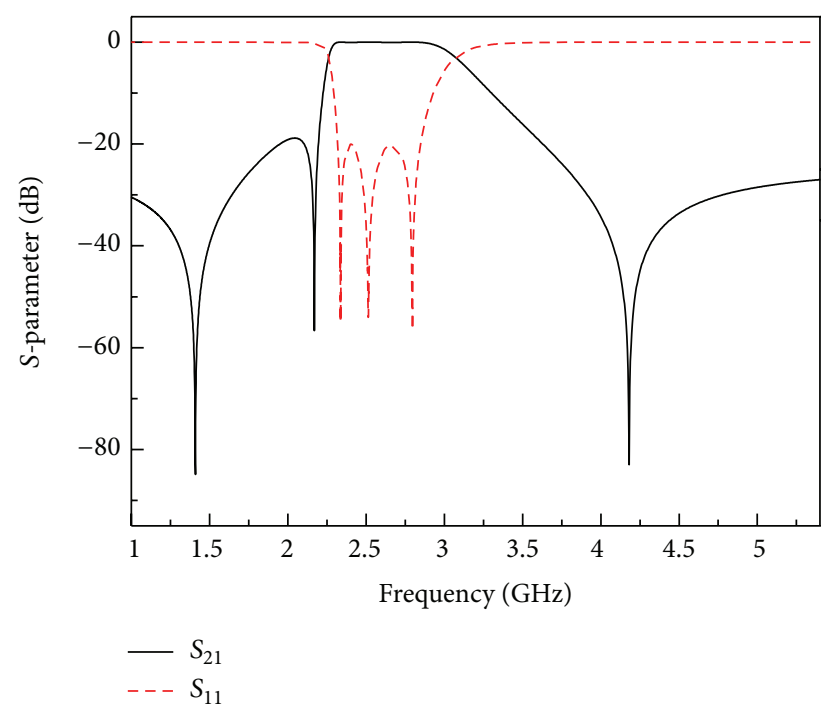

FIGURE 3: Synthesized theory result of triple-mode BPF.

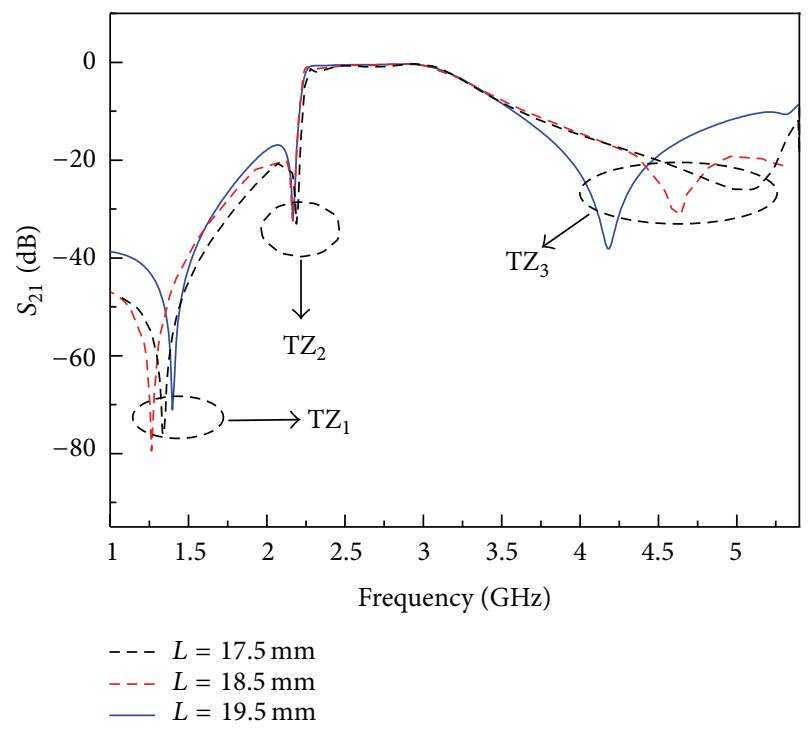

FIgURE 4: The changes of transmission zeros versus $L$.

$L$ increases, that is, the coupling gap between input line and output line decreases, the coupling between source and load increases accordingly. Consequently, the third transmission zero $\left(\mathrm{TZ}_{3}\right)$ moves towards the passband, whereas the other two transmission zeros almost remain unchanged. Thus, it turns out to be convenient to realize filters with sharp transition band. Simultaneously, increment of the length of microstrip feed line may increase the coupling between source/load and resonators, and the transmission zero $\left(\mathrm{TZ}_{1}\right)$ will shift to the passband.

To study the influence of the microstrip resonator on the characteristic of the filter, frequency responses of triple-mode BPF and dual-mode resonator are compared in Figure 5. The dotted curve indicates the transmission characteristic of DGS dual-mode resonator and the solid line indicates the transmission characteristic of proposed structure. It is

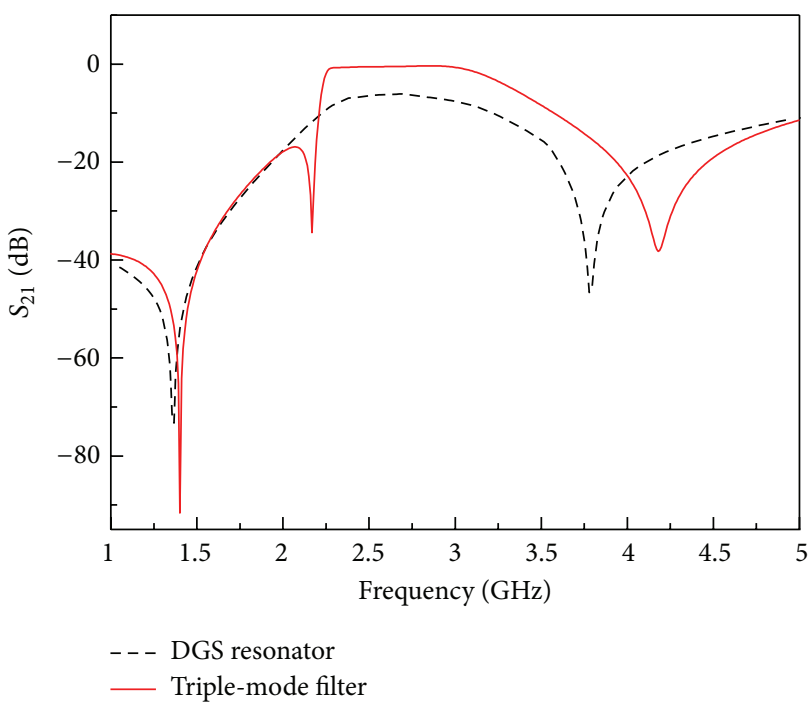

FIGURE 5: The comparison of frequency responses between triplemode BPF and dual-mode resonator.

clearly observed that an additional transmission zero is created at about $2.17 \mathrm{GHz}$ when the microstrip open-loop resonator is loaded. The phenomenon can be explained by the fact that the microstrip open-loop resonator adds an extra transmission path to the circuit, and it will counteract with signal from another path at certain frequency. In addition, the first transmission zero $\mathrm{TZ}_{1}$ almost keeps stable and the transmission zero in the upper stopband of the filter shifts away from the pass band, when the microstrip open-loop resonator is added to the circuit.

Figure 6 gives the simulated transmission characteristics of the filter versus $L_{8}$. When $L_{8}$ increases from $4.5 \mathrm{~mm}$ to $5.5 \mathrm{~mm}, \mathrm{TZ}_{2}$ moves towards lower frequency and $\mathrm{TZ}_{3}$ shifts to higher frequency, while $\mathrm{TZ}_{1}$ seems to keep unchanged. When $L_{8}$ increases from $5.5 \mathrm{~mm}$ to $6.5 \mathrm{~mm}$, three transmission zeros shift accordingly. It is obvious that the bandwidth of the filter will enlarge with the increment of $L_{8}$. Changing $L_{8}$ will modify the overlapping length between feed line and microstrip resonator, and the coupling between source/load and microstrip resonator will vary accordingly. Moreover, resonant frequency of the microstrip resonator will change with $L_{8}$, so that $L_{8}$ influences not only the bandwidth of the filter but also the location of the transmission zeros.

\section{Simulation and Experimental Results}

For the sake of validating above-mentioned theory, a compact and high selectivity triple-mode BPF is designed and fabricated. The designed filter has a central frequency of $2.57 \mathrm{GHz}$ and fractional bandwidth of $15 \%$ with equal ripple of $0.0432 \mathrm{~dB}$. A substrate with a relative dielectric constant of 3.5 and a thickness of $0.8 \mathrm{~mm}$ is used in the design. Obtained parameters of the filter shown in Figure 1 are as follows: $L$ $=19.5 \mathrm{~mm}, L_{1}=15 \mathrm{~mm}, L_{2}=3 \mathrm{~mm}, L_{3}=1.8 \mathrm{~mm}$, $L_{4}=1 \mathrm{~mm}, L_{5}=5.5 \mathrm{~mm}, L_{6}=3 \mathrm{~mm}, L_{7}=16 \mathrm{~mm}$, 


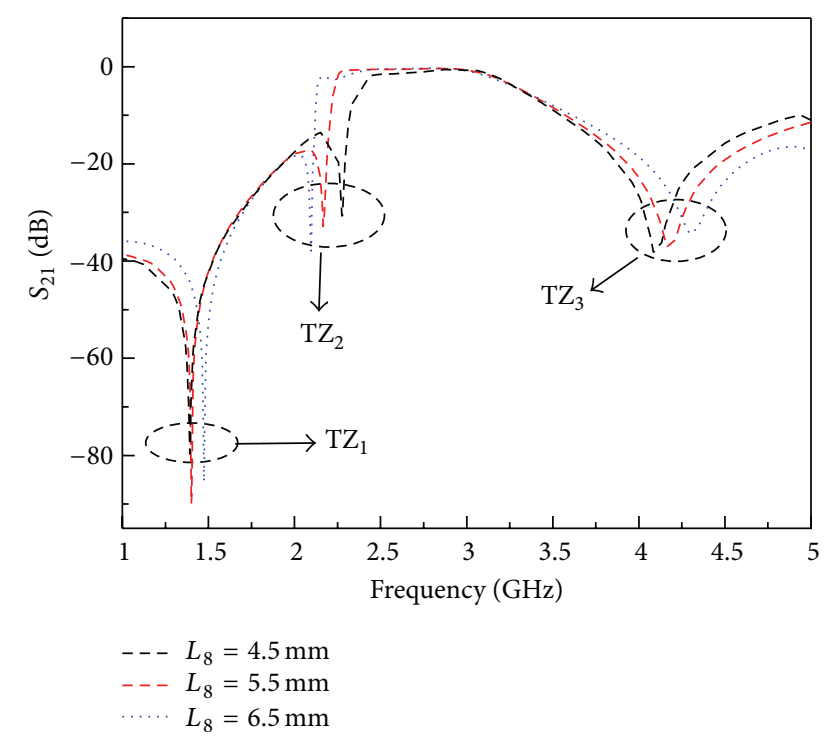

FIgURE 6: Transmission characteristics of the filter versus $L_{8}$.

$L_{8}=5.5 \mathrm{~mm}, L_{9}=2.6 \mathrm{~mm}, L_{10}=18 \mathrm{~mm}, W=1.5 \mathrm{~mm}$, $W_{1}=0.5 \mathrm{~mm}, W_{2}=0.5 \mathrm{~mm}$, and $W_{3}=1 \mathrm{~mm}$. The filtering performance is measured by using Network Analyzer AV3926, and a comparison between EM simulated results and measured results is shown in Figure 7. Solid lines and dotted lines indicate the simulated and measured results, respectively. The passband of the proposed filter is from $2.2 \mathrm{GHz}$ to $3.68 \mathrm{GHz}$, and its passband return loss is larger than $20 \mathrm{~dB}$. Three transmission poles are clearly observed at $2.31 \mathrm{GHz}, 2.5 \mathrm{GHz}$, and $2.82 \mathrm{GHz}$ in the passband of the filter. Three transmission zeros are generated at $1.48 \mathrm{GHz}$, $2.17 \mathrm{GHz}$, and $4.18 \mathrm{GHz}$, which improve the selectivity of the filter. The simulated and measured maximum insertion loss in the passband are $1 \mathrm{~dB}$ and $1.12 \mathrm{~dB}$, respectively. Apart from the frequency shift that may be caused by the discrepancy of the dielectric constant between its nominal value and real value, measured results agree well with the simulated results. The photograph of the fabricated filter is shown in Figure 8. The designed filter circuit occupies the overall size of about $30 \mathrm{~mm} \times 15 \mathrm{~mm}$.

\section{Conclusion}

A novel miniature microstrip triple-mode bandpass filter is proposed in this paper. Three modes are obtained by combination of a dual-mode DGS resonator and a microstrip resonator. The coupling matrix of proposed structure is established to further explain the proposed design. Three transmission zeros are realized in the stopband of the filter, which greatly improve the selectivity and attenuation of proposed filter. Measured results agree well with the simulated results, verifying the proposed structure and design methodology.

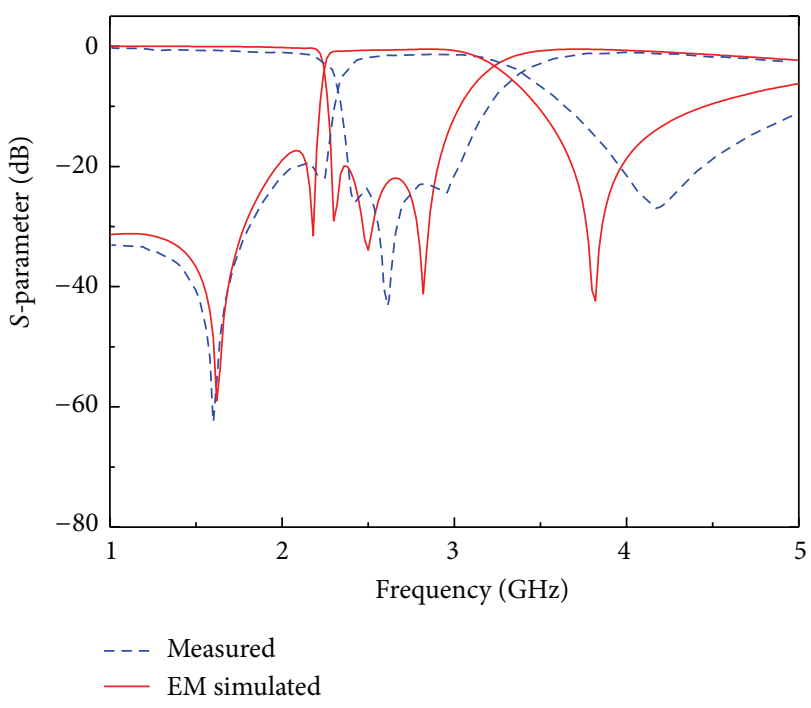

FIGURE 7: Comparison between EM simulated and measured results of the proposed filter.

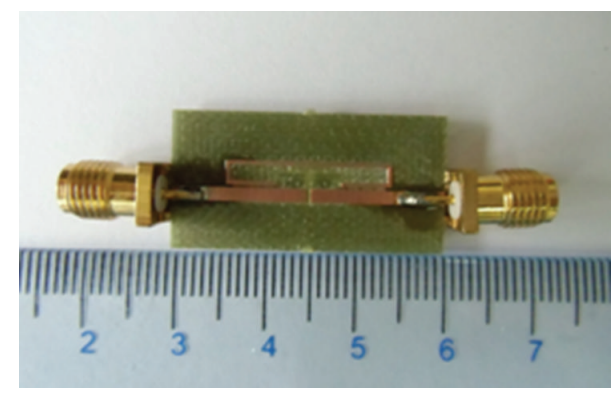

(a)

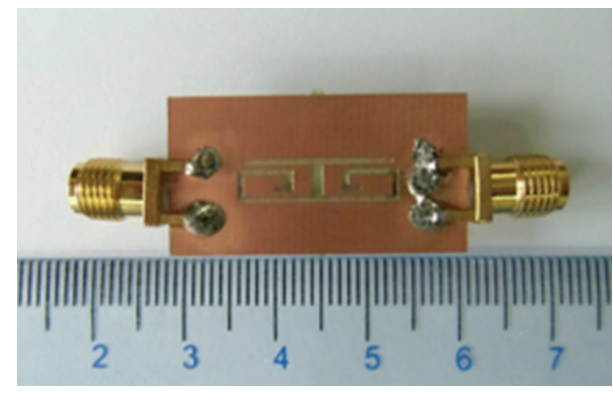

(b)

Figure 8: Photographs of the fabricated filter: (a) top view and (b) bottom view.

\section{Acknowledgments}

This work is supported by National Science Foundation Committee of China (no. 61061001, no. 61161005), Open Fund from State Key Laboratory of Millimeter Wave (K201210), Training Program for Young Scientists of Jiangxi Province, and Natural Science Foundation of Jiangxi Province (20114BAB211011). 


\section{References}

[1] S. W. Ren, H. L. Peng, J. F. Mao, and A. M. Gao, "Compact quasielliptic wideband bandpass filter using cross-coupled multiplemode resonator," IEEE Microwave and Wireless Components Letters, vol. 22, no. 8, pp. 397-399, 2012.

[2] I. T. H. Wolff, "Microstrip bandpass filter using degenerate modes of a microstrip ring resonator," Electronics Letters, vol. 8, no. 12, pp. 302-303, 1972.

[3] L. Zhu, P. M. Wecowski, and K. Wu, "New planar dual-mode filter using cross-slotted patch resonator for simultaneous size and loss reduction," IEEE Transactions on Microwave Theory and Techniques, vol. 47, no. 5, pp. 650-654, 1999.

[4] J. S. Hong and M. J. Lancaster, "Bandpass characteristics of new dual-mode microstrip square loop resonators," Electronics Letters, vol. 31, no. 11, pp. 891-892, 1995.

[5] R. Wu and S. Amari, "New triangular microstrip loop resonators for bandpass dual-mode filter applications," in Proceedings of the IEEE MTT-S International Microwave Symposium, pp. 941-944, June 2005.

[6] R. J. Mao and X. H. Tang, "Novel dual-mode bandpass filters using hexagonal loop resonators," IEEE Transactions on Microwave Theory and Techniques, vol. 54, no. 9, pp. 3526-3533, 2006.

[7] J. S. Hong and H. Shaman, "Dual-mode microstrip open-loop resonators and filters," IEEE Transactions on Microwave Theory and Techniques, vol. 55, no. 8, pp. 2099-2109, 1996.

[8] Z. Q. Cheng, J. Li, X. G. Mao, S. Tan, and J. K. Chen, "Flip-chip integrated oscillator with reduced phase noise and enhanced output power by using DGS," Journal of Infrared and Millimeter Waves, vol. 27, no. 6, pp. 401-404, 2008.

[9] A. Boutejdar, A. El-Sherbini, and A. S. Omar, "Method for widening the reject-band in lowpass/bandpass filters by employing coupled C-shaped defected ground structure (DGS)," IET Microwaves, Antennas \& Propagation, vol. 17, no. 12, pp. 1405-1408, 2008.

[10] A. Boutejdar, A. Elsherbini, A. Balalem, J. Machac, and A. S. Omar, "Design of new DGS hairpin microstrip bandpass filter using coupling matrix method," in Progress in Electromagnetics Research Symposium, pp. 261-265, Prague, Czech Republic, 2007.

[11] W. Feng, K. Deng, W. Che, and S. Dong, "Novel microstrip bandpass filter based on defected ground structure and slotline coupling techniques," Microwave and Optical Technology Letters, vol. 52, no. 1, pp. 4-6, 2010.

[12] A. Mohan and A. Biswas, "Dual-band bandpass filter using defected ground structure," Microwave and Optical Technology Letters, vol. 51, no. 2, pp. 475-479, 2009.

[13] B. Wu, C. H. Liang, P. Y. Qin, and Q. Li, "Compact dualband filter using defected stepped impedance resonator," IEEE Microwave and Wireless Components Letters, vol. 18, no. 10, pp. 674-676, 2008.

[14] Z. Ma, W. He, C. P. Chen, Y. Kobayashi, and T. Anada, "A novel compact ultra-wideband bandpass filter using microstrip stubloaded dual-mode resonator doublets," in Proceedings of the IEEE MTT-S International Microwave Symposium Digest (MTT '08), pp. 435-438, Atlanta, Ga, USA, June 2008.

[15] X. Guan, X. Wang, B. Wang, Y. Yuan, and H. W. Liu, "Design and implementation of compact hybrid four-mode bandpass filter with multi-transmission zeros," Progress in Electromagnetics Research Letters, vol. 34, pp. 65-74, 2012.
[16] X. Guan, W. Fu, H.-W. Liu, D. Ahn, and J.-S. Lim, "A novel dualmode bandpass filter based on a defected waveguide resonator," ETRI Journal, vol. 33, no. 6, pp. 953-956, 2011.

[17] S. Amari, U. Rosenberg, and J. Bornemann, "Adaptive synthesis and design of resonator filters with source/load-multiresonator coupling," IEEE Transactions on Microwave Theory and Techniques, vol. 50, no. 8, pp. 1969-1978, 2002. 

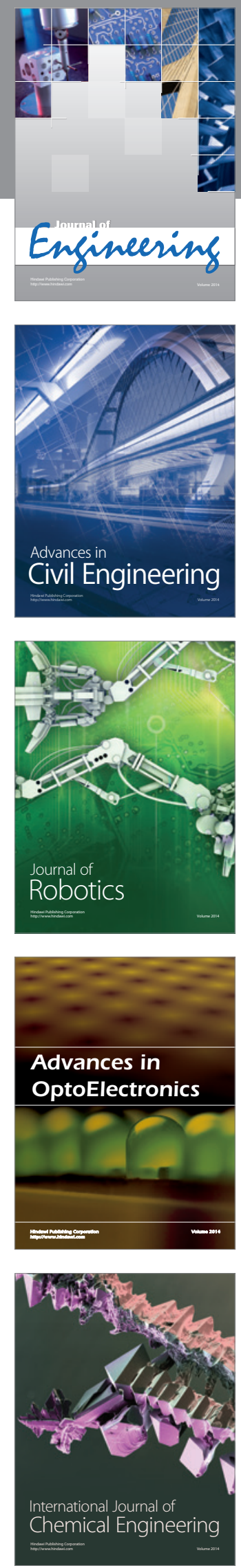

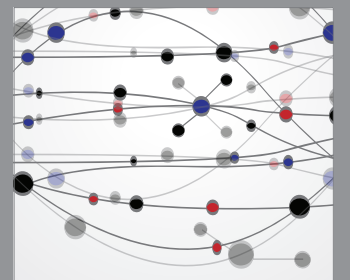

The Scientific World Journal
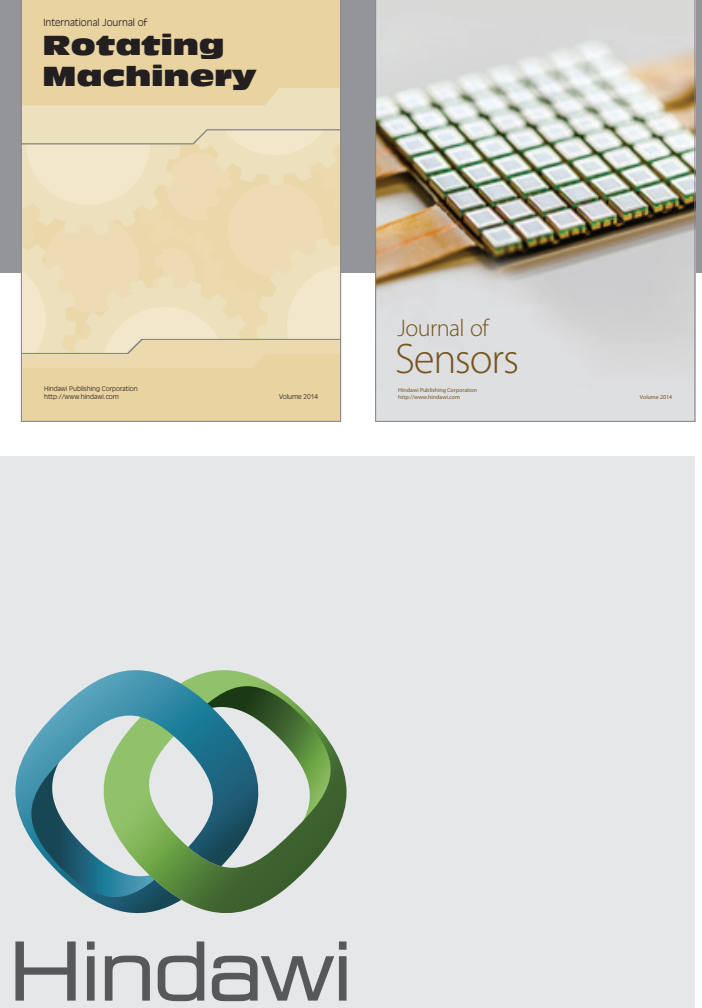

Submit your manuscripts at http://www.hindawi.com
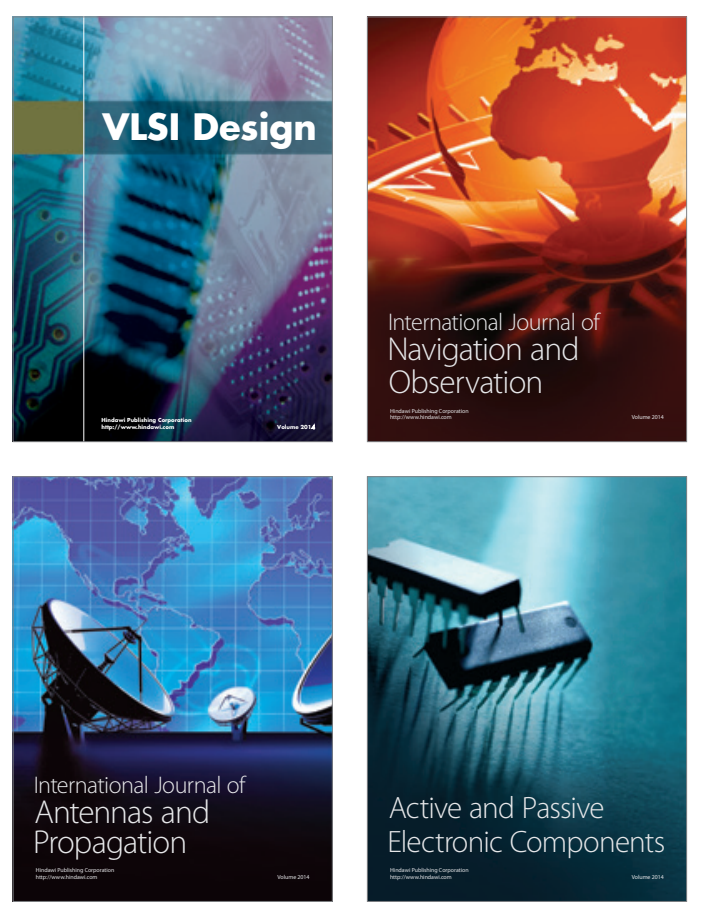
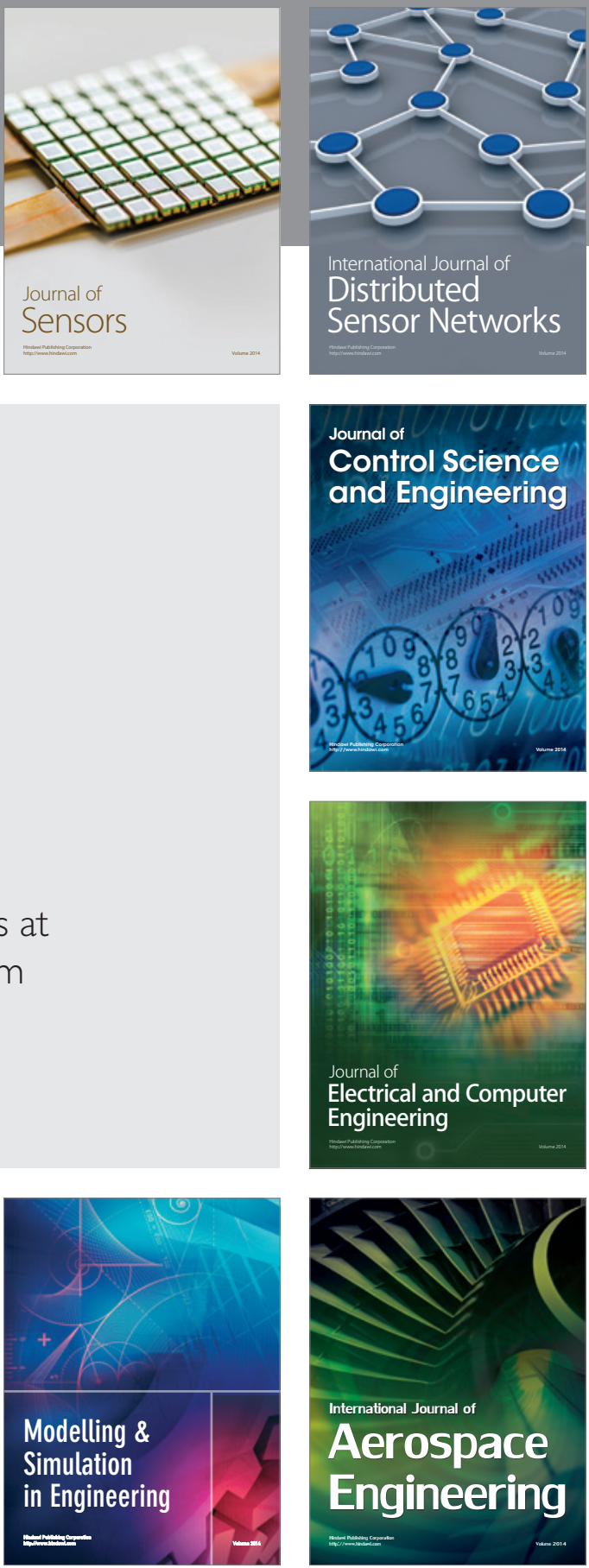

Journal of

Control Science

and Engineering
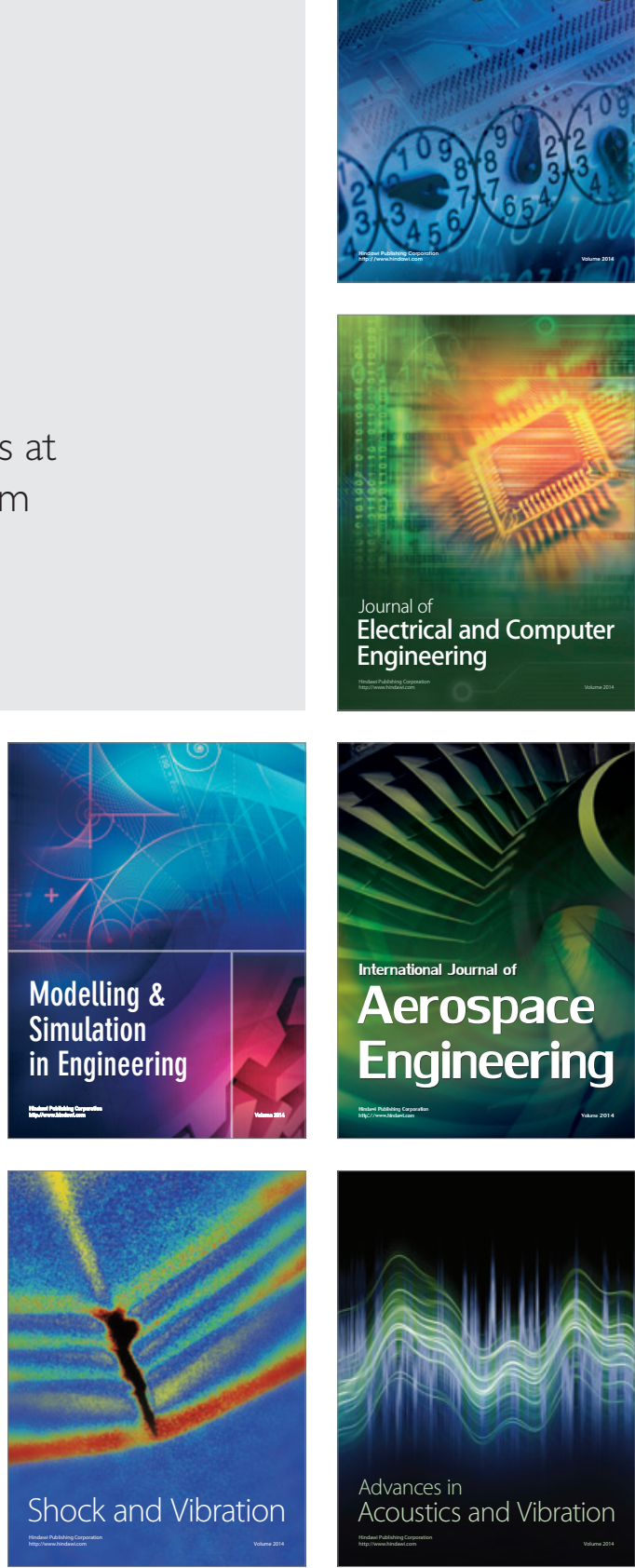